\title{
Design and Evaluation of a Fiber-Optic Grip Force Sensor with Compliant 3D-Printable Structure for (f)MRI Applications
}

\author{
Tobias L. Bützer, ${ }^{1}$ Mike D. Rinderknecht, ${ }^{1}$ Gunda H. Johannes, ${ }^{1}$ Werner L. Popp, ${ }^{1,2}$ \\ Rea Lehner, ${ }^{3}$ Olivier Lambercy, ${ }^{1}$ and Roger Gassert ${ }^{1}$ \\ ${ }^{1}$ Rehabilitation Engineering Laboratory, ETH Zurich, 8092 Zurich, Switzerland \\ ${ }^{2}$ Spinal Cord Injury Center, Balgrist University Hospital, 8008 Zurich, Switzerland \\ ${ }^{3}$ Neural Control of Movement Lab, ETH Zurich, 8057 Zurich, Switzerland \\ Correspondence should be addressed to Tobias L. Bützer; tobias.buetzer@hest.ethz.ch
}

Received 18 March 2016; Accepted 31 May 2016

Academic Editor: Paola Saccomandi

Copyright (C) 2016 Tobias L. Bützer et al. This is an open access article distributed under the Creative Commons Attribution License, which permits unrestricted use, distribution, and reproduction in any medium, provided the original work is properly cited.

\begin{abstract}
Grip force sensors compatible with magnetic resonance imaging (MRI) are used in human motor control and decision-making research, providing objective and sensitive behavioral outcome measures. Commercial sensors are expensive, cover limited force ranges, rely on pneumatic force transmission that cannot detect fast force changes, or are electrically active, which increases the risk of electromagnetic interference. We present the design and evaluation of a low-cost, 3D-printed, inherently MRI-compatible grip force sensor based on a commercial intensity-based fiber-optic sensor. A compliant monobloc structure with flexible hinges transduces grip force to a linear displacement captured by the fiber-optic sensor. The structure can easily be adapted for different force ranges by changing the hinge thickness. A prototype designed for forces up to $800 \mathrm{~N}$ was manufactured and showed a highly linear behavior (nonlinearity of 2.37\%) and an accuracy of $1.57 \%$ in a range between zero and $500 \mathrm{~N}$. It can be printed and assembled within one day and for less than $\$ 300$. Accurate performance was confirmed, both inside and outside a 3 T MRI scanner within a pilot study. Given its simple design allowing for customization of sensing properties and ergonomics for different applications and requirements, the proposed grip force handle offers researchers a valuable scientific tool.
\end{abstract}

\section{Introduction}

Since its introduction to the research community in the early 1990s [1], functional magnetic resonance imaging (fMRI) has continuously improved its standing to where it is today-the most popular neuroimaging tool in human neuroscience. Of particular convenience are its noninvasiveness, high spatial resolution, and ability to gauge brain activity in areas that lie below the surface of the cortex.

Human hand motor control and its central nervous regulation are explored in multiple research fields, such as the investigation of neural mechanisms underlying movement in the healthy brain [2] or effects on these mechanisms by age [3] and disease [4] and the rehabilitation of function after injury [5]. Researchers have further employed grip force as a quantitative outcome measure of subjective values (e.g., effort discounting [6]). A subject can fairly easily provide responses of varying value estimations by producing variable grip force amplitudes within the constraints of the MRI environment. More recently, attempts have been made to identify neural correlates of such internal value representations in fMRI studies utilizing grip force as outcome measure [7].

The broad scope of application of force sensing devices in the scientific investigation of movement control underlines a need for MRI-compatible force transducers that can measure the full range of human voluntary grip force in a controlled and reproducible manner. Due to its unique and challenging environment involving strong (mostly 1.5 or $3 \mathrm{~T}$ ) static and switching magnetic fields as well as radiofrequency pulses and a supine subject instructed to move as little as possible, there are specific constraints to be considered with regard to the design and fabrication of a force sensor for fMRI studies to ensure its safe and proper functioning (see also [8]). In short, an MRI-compatible grip force device should neither affect 
the imaging nor be affected by the magnetic field of the scanner. For detailed guidelines and design criteria for the development of a broad spectrum of MRI-compatible devices, see [9]. Various commercial grip force sensors are available at affordable prices, for example, from ADInstruments (Sidney, Australia) or Vernier (Beaverton, OR, USA). Yet, these cannot be used in an MRI environment, as they are largely constructed out of ferromagnetic parts and transmit signals via electric cables.

Force sensors taking into account the electromagnetic and spatial constraints of an MRI scanner have been developed (an overview on such systems is provided in [10]), but only few commercial MRI-compatible grip force sensors, such as the Biopac Systems pump bulb pressure transducer, exist. They are expensive (several thousand \$) and often rely on air pressure, which limits the transmission of rapidly changing forces as a result of air compressibility in the tube. In research, different principles have been used to measure grip force in the MRI environment. Often, these systems rely on nonmagnetic but metallic and electrically active sensors $[11,12]$ or on sensors that measure pressure via hydrostatic transmission [13]. The former carry the risk of eddy currents and therefore image-disturbing thermal and mechanical effects as well as electromagnetic interference, while the latter introduce measurement errors due to friction in the transmission from grip force to hydrostatic pressure [13]. Most MRIcompatible force sensors, however, use compliant structures to transform force into deformation, which is measured either by load cells attached to the probe $[11,12,14]$ or by fiberoptic sensors [15-18]. Due to advances in development and availability, fiber-optic sensors have received increasing interest (for an overview, refer to [19]), as they are inherently compatible with the harsh but sensitive MR environment. Within the field of fiber-optic sensors, different principles such as time-of-flight, interferometric, or Doppler effect sensing are commonly used [20]. Owing to their simplicity and therefore affordable price coupled with high performance, intensitybased sensors are widely found in recent research [8, 20-22].

The compliant structures used with fiber-optic based force sensing devices are mostly made of nonmagnetic metals (e.g., aluminum $[21])$ or polymers $([17,18])$. But only very few devices which combine fiber-optic sensors with nonmetallic compliant structures to measure grip force have been presented (Riener et al. [23] and Force Transducers, Neuroimaging Solutions, Gainesville, FL). Recently, the development and availability of $3 \mathrm{D}$ printers offered new possibilities for manufacturing nonmetallic compliant structures that can easily be customized to accommodate for different sensing ranges, ergonomic aspects, and applications. Force sensor designs using rapid prototyping have been proposed, but so far only for low force ranges (i.e., under $10 \mathrm{~N}$ ) [24-26]. For MRI-compatible grip force measurement, forces ranging up to $800 \mathrm{~N}$ (95\% percentile of grip strength for males is $729 \mathrm{~N}$ [27]) have to be measured. Therefore, there is a need for new design principles for 3D-printed fiber-optic based grip force sensors that accommodate higher force ranges.

In the present paper, we describe and discuss the design and characterization of a low-cost, inherently MRI-compatible grip force sensor which is based on a commercially

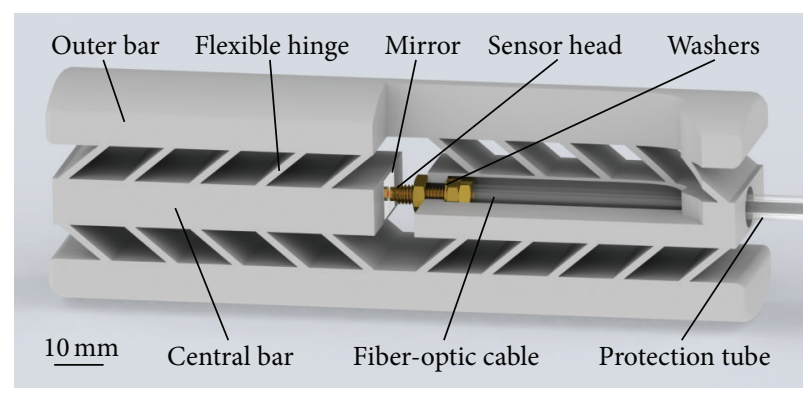

FIGURE 1: Rendering of the developed grip force sensor with a partial cut in the monobloc structure to visualize the placement of the fiberoptic sensor and the mirror.

available intensity-based fiber-optic sensor and a custom compliant structure, printed on a low-cost $3 \mathrm{D}$ printer. The presented sensor is capable of measuring grip forces up to $800 \mathrm{~N}$. We begin by describing its design and report results of a finite element method (FEM) analysis of the compliant structure of the sensor, including the design parameters that should be adjusted for different sensing ranges. Subsequently, we elaborate on a full characterization conducted on the manufactured device. Finally, we report a pilot study where the grip force sensor is used in a typical experimental condition of an fMRI setup, illustrating its inherent MRI compatibility.

\section{Materials and Methods}

2.1. Concept and Materials. The concept of the grip force sensor is based on a 3D-printable monobloc structure (handle) with integrated flexible hinges, which is ergonomic and can be held with a power grip. A $3 \mathrm{D}$ rendering of the design can be seen in Figure 1. By enclosing the handle, the external grip force is applied on the two outer bars of the handle, which distribute the force along an array of flexible hinges. As a result of this force, the flexible hinges deform, and the distance between the two central bars increases and can be measured by the fiber-optic sensor, as it is mounted on one side and the mirror reflecting the light beam on the other. As the two central bars-and therefore sensor head and the mirror-move away from each other when the grip force sensor is loaded, no contact and subsequent damage of these elements are possible.

An affordable commercial fiber-optic sensor system (brass sensor head FUE 999C1004 and analog amplifier FWDK 10U84Y0, Baumer Electric, Frauenfeld, Switzerland, $\$ 260$ ) was used for the grip force sensor. This system has already been successfully employed in earlier related work [8, $21,24]$. The fiber-optic sensor works according to the reflected light intensity measurement concept (see Figure 2) using a pulsed red LED (680 nm wavelength) as light source. The sensor head and cable consist of two fiber-optic channels. One channel emits light, which is reflected on the mirror placed on the central bar of the compliant structure, whereas the second channel collects a fraction of the reflected light (dependent on the distance between the sensor head and the mirror). According to characterization of the fiber-optic sensor by 


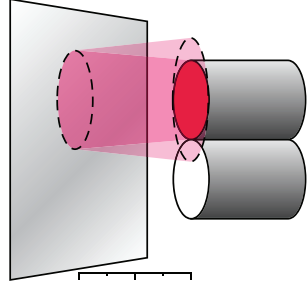

(a)

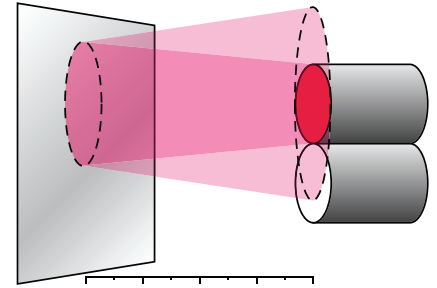

(b)
FIGURE 2: Concept of the reflected light intensity measurement. (a) The upper fiber-optic channel emits the light which is reflected on the mirror. The lower fiber-optic channel collects a fraction of the reflected light. (b) If the mirror is placed at a larger distance, the second fiber-optic channel collects more light.

Chapuis et al., the distance range for optimal operation, that is, highest linearity and sensitivity, is from zero to $0.6 \mathrm{~mm}$ [8]. Therefore, a precise positioning of the sensor head with respect to the mirror is required. In our design, this can be achieved by adjusting the sensor head position by simply adding thin brass washers (which vary in thickness by $0.1 \mathrm{~mm}$ ) between the 3D-printed structure and the nut/ locking nut. Using this method, the distance between mirror and sensor head can be adjusted to $0.1 \mathrm{~mm}$ when no grip force is applied, to avoid contact between the two elements. By taking an optimal operation range from 0.1 to $0.6 \mathrm{~mm}$ into account, the compliant structure can be dimensioned for the desired force range. The amplifier is not MRI-compatible. Therefore, it is located outside of the MRI-scanner room and connected to the sensor head via a $10 \mathrm{~m}$ fiber-optic cable. The analog voltage provided by the amplifier can be acquired by a data acquisition card.

The compliant structure was made of PLA (polylactic acid, an MRI-compatible, biodegradable thermoplastic, electric volume resistivity $\rho=5 \cdot 10^{18} \mathrm{Ohm} \mathrm{cm} \mathrm{[28],} \mathrm{Young's}$ modulus $E=2400 \mathrm{MPa}$, and yield stress $\sigma_{\text {yield }}=25 \mathrm{MPa}$ [29]) and fabricated with a 3D printer (Ultimaker 2 with $0.4 \mathrm{~mm}$ nozzle, Ultimaker B.V., Geldermalsen, Netherlands) using fused deposition modeling (FDM), that is, layer-by-layer extrusion. This technology not only enables fast and affordable production of compliant probes but also allows in combination with CAD software adapting the design to the requirements of the application, for example, in terms of handle dimensions and force measurement range (see Section 2.3). It should be noted that the material properties of PLA are strongly temperature dependent but do not vary significantly up to $50^{\circ} \mathrm{C}[29]$.

2.2. Theoretical Model of Structure Deformation. The transversal compression of the grip force sensor due to the applied force $F_{\text {Grip }}$ will lead to a longitudinal outward displacement $\Delta f$ for each of the two central elements, as schematically represented in Figure 3(a). For the theoretical model we assume that $F_{\text {Grip }}$ is uniformly distributed on the $n_{\text {Hinges }}$ equally spaced flexible hinges resulting in $F_{\text {Hinge }}=\left(1 / n_{\text {Hinges }}\right) F_{\text {Grip }}$ per hinge.
With this assumption a model can be derived for an individual hinge. According to Henein [30], the relation between any shear force $F$ and resulting displacement $\Delta f$ in a flexible hinge clamped on either side is $\Delta f=F / K$, where $K=12 E I / l^{3}$ and $I=b h^{3} / 12$, with $K$ being the rigidity, $E$ the Young's modulus, $I$ the moment of inertia, and $l, b$, and $h$ the length, width, and thickness of the flexible hinge, respectively (see Figure 3(c)). Since in our design the flexible hinge is at an initial angle $\alpha$ (see Figure 3(b)), the responsible force for the deflection of the hinge is $F_{\perp}=\cos (\alpha) F_{\text {Hinge }}$. Assuming a linear behavior of the resulting displacement due to very small motion, the total deflection of the hinge can be written as follows:

$$
\Delta f_{\perp}=\frac{\cos (\alpha) F_{\text {Hinge }}}{K} .
$$

Yet, only the deflection $\Delta f=\sin (\alpha) \Delta f_{\perp}$ in longitudinal direction results in a change of distance between the mirror and the sensor head (see Figures 3(a) and 3(b)). Therefore, for the deflection of interest, (1) leads to

$$
\Delta f=\frac{\sin (\alpha) \cos (\alpha) F_{\text {Hinge }}}{K}
$$

or including the dimensions of the flexible hinge

$$
\Delta f=\frac{\sin (\alpha) \cos (\alpha) l^{3} F_{\text {Hinge }}}{b h^{3} E} .
$$

For all ten hinges the total amount of displacement between the mirror and the sensor head (two times $\Delta f$ ) as a function of $F_{\text {Grip }}$ results in

$$
2 \Delta f=\frac{2 \sin (\alpha) \cos (\alpha) l^{3}}{n_{\text {Hinges }} b h^{3} E} F_{\text {Grip }} .
$$

It should be noted that the sensitivity of the flexible hinges to parasitic forces is proportional to $(h / l)^{2}$ and $(h / b)^{2}$ [30]. Therefore, these ratios should be kept low to minimize the effects of parasitic forces.

2.3. Dimensioning and Finite Element Model Analysis of Deformation and Stress. Using finite element method (FEM) in SolidWorks 2015 (Dassault Systèmes, Vélizy-Villacoublay, France), several grip force sensor designs were iteratively tested to match the order of magnitude of the desired force range. The ergonomic aspect limiting the outside dimensions was tested using styrofoam models and subjects with different hand sizes. The final design included ten hinges on each side and had the following dimensions: total length of $140 \mathrm{~mm}$, height of $45.4 \mathrm{~mm}$ (with a curved surface of radius $22.7 \mathrm{~mm}$ ), and width of $28 \mathrm{~mm}$ (which corresponds directly to the width of the hinges $b$ ). Since the outer and central bars of the grip force sensor require a certain thickness as they should not deform and have to house the fiber-optic sensor, the design resulted in a hinge length $l$ of $11 \mathrm{~mm}$ at an angle $\alpha$ of $40^{\circ}$. The remaining free parameter is the hinge thickness $h$, which can be customized for different force ranges. The proposed compliant structure was analyzed using FEM in order to 


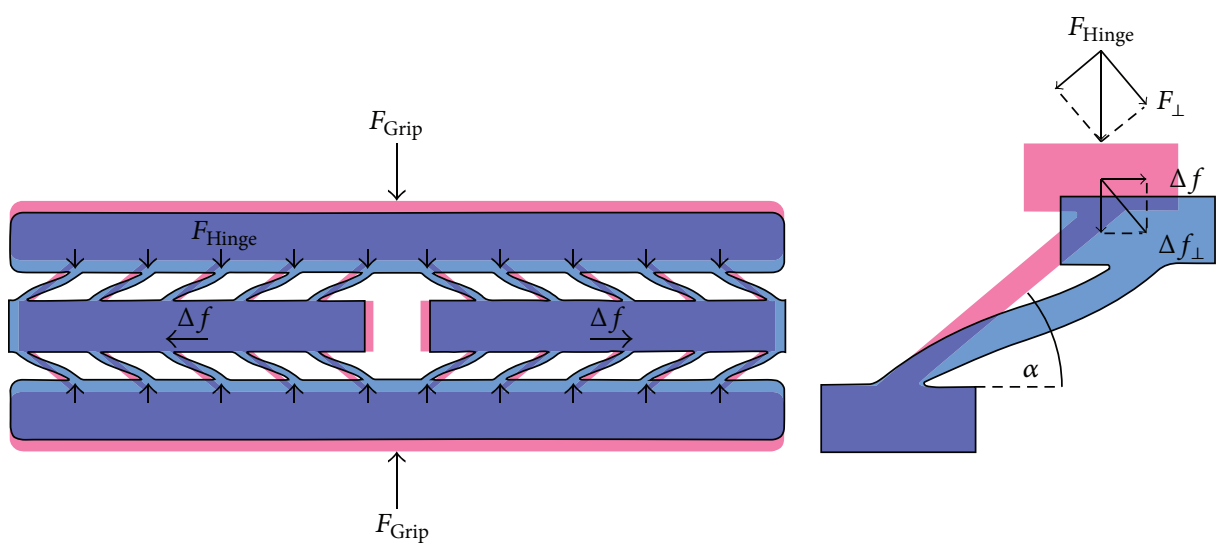

(a)

(b)

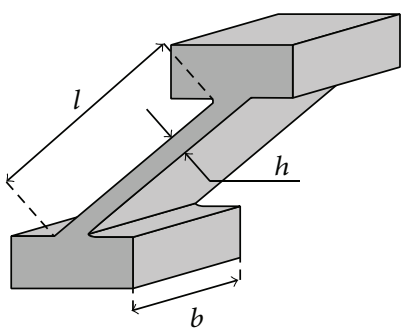

(c)

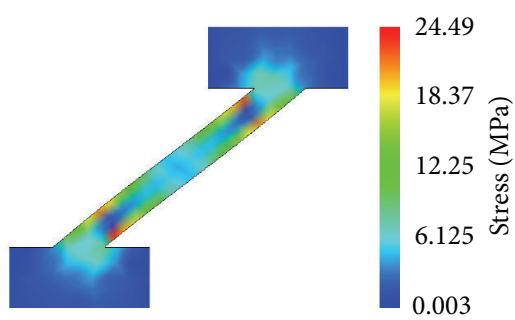

(d)

FIGURE 3: (a) Red: flexible structure before deformation due to the applied grip force $F_{\text {Grip }}$. Blue: deformed structure when $F_{\text {Grip }}$ is applied. (b) Detailed view of an individual flexible hinge with the forces acting upon it and the resulting deflections. (c) Hinge parameters: length $l$, width $b$, and thickness $h$ of the flexible hinge. (d) Finite element model (FEM) analysis of the internal stress in a hinge when applying $F_{\text {Grip }}=800 \mathrm{~N}$ for a hinge thickness of $h=1.4 \mathrm{~mm}$.

(i) calculate the stress distribution in the flexible hinges, (ii) explore possible force ranges and corresponding hinge parameters for scaling and customization of the grip force sensor, and (iii) find the optimal hinge thickness for our application.

As in the theoretical model described in Section 2.2, an equally distributed grip force was assumed. A set of different grip forces $F_{\text {Grip }}$ ranging from $200 \mathrm{~N}$ to $800 \mathrm{~N}$ in steps of $200 \mathrm{~N}$ was simulated, as the maximal voluntary grip force accounting for $95 \%$ of the male population goes up to $729 \mathrm{~N}$ [27]. As a second varying parameter, the hinge thickness was simulated for the following set: $\in\{1.2,1.3,1.4,1.5,1.6\} \mathrm{mm}$. Both the resulting total displacement $2 \Delta f\left(F_{\text {Grip }}, h\right)$ and the maximum stress in the compliant structure $\sigma_{\text {max }}\left(F_{\text {Grip }}, h\right)$ were simulated. By fitting $2 \mathrm{D}$ parametric functions to the simulated FEM data as a function of grip force $F_{\text {Grip }}$ and hinge thickness $h$, the displacement and stress can be numerically described:

$$
\begin{aligned}
2 \Delta f\left(F_{\text {Grip }}, h\right) & =\frac{0.001443 F_{\text {Grip }}}{h^{2.882}}, \\
\sigma_{\max }\left(F_{\text {Grip }}, h\right) & =\frac{0.05838 F_{\text {Grip }}}{h^{1.863}},
\end{aligned}
$$

where the parameters $F_{\text {Grip }}$ and $h$ are in $\mathrm{N}$ and mm, respectively, the resulting $2 \Delta f$ is in $\mathrm{mm}$, and the stress $\sigma_{\max }$ in
MPa. $r^{2}$ of both fits were larger than 0.9997 indicating that the proportional relationship between the displacement and the grip force in the theoretical model described in Section 2.2, as well as the underlying assumption of linear behavior of the hinge displacement due to the very small motion, can be validated, as both parametric functions are highly linear with respect to the grip force $F_{\text {Grip }}$ for any given hinge thickness $h$.

In order to explore the possible design parameter ranges to customize the grip force sensor, two constraints were defined: $2 \Delta f \leq 0.5 \mathrm{~mm}$ (as the optimal sensing range of the fiber-optic sensor is $0-0.6 \mathrm{~mm}$ and at no applied force the distance between sensor head and mirror is $0.1 \mathrm{~mm}$ ) and $\sigma_{\max } \leq 25 \mathrm{MPa}$ (below the yield point $\sigma_{\text {yield }}$, i.e., to avoid plastic deformations of the PLA material). The shaded areas in Figure 4 show the thickness-force combinations where the constraints cannot be fulfilled. The ideal design would allow for the highest maximum force, that is, $800 \mathrm{~N}$, and the minimum hinge thickness resulting in the largest displacements to increase the signal-to-noise ratio (SNR) of the sensor output. For the desired force range of $800 \mathrm{~N}$ the allowed minimal (and therefore optimal) hinge thickness $\mathrm{h}$ is $1.4 \mathrm{~mm}$ (indicated in Figure 4 by a black circle).

Using a grip force $F_{\text {Grip }}=800 \mathrm{~N}$ and a hinge thickness $h=$ $1.4 \mathrm{~mm}$, the maximum internal stress in the compliant structure $\sigma_{\max }$ was $24.49 \mathrm{MPa}$ and the stress distribution in one 


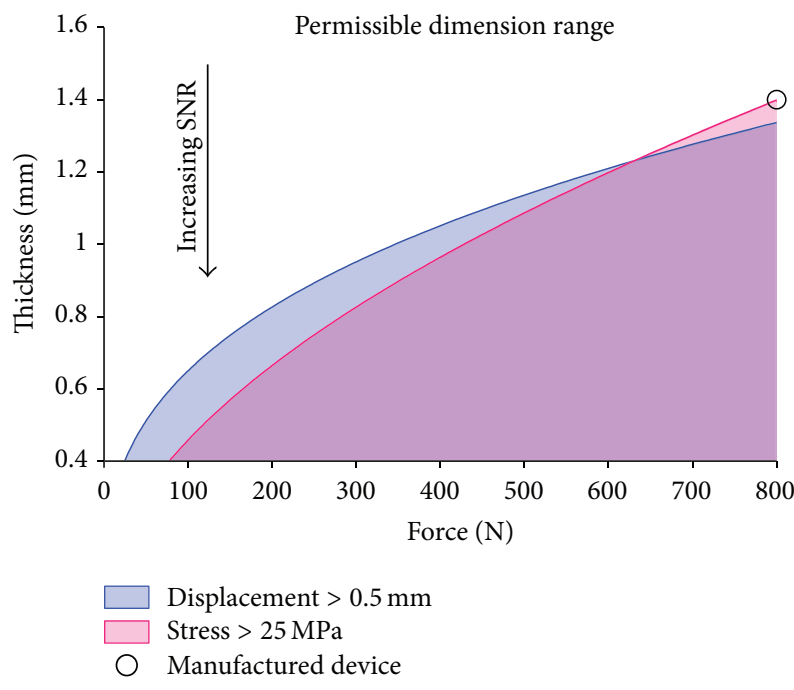

Figure 4: Permissible dimension range (white) depending on grip force $F_{\text {Grip }}$ and hinge thickness $h$. The shaded areas represent the areas, where the working point of the fiber-optic sensor enters the nonlinear region due to a too large displacement or where the internal stress in the compliant structure increases beyond yield point. A lower hinge thickness results in a better signal-to-noise ratio (SNR), as the sensor output voltage range is increased due to larger displacements. The hinge thickness for the manufactured device (black circle) was chosen based on a maximal SNR for a desired force range of $800 \mathrm{~N}$.

hinge is visualized in Figure 3(d). The FEM stress analysis in the different hinges was in accordance with our hypothesis that the grip force $F_{\text {Grip }}$ gets equally distributed to all hinges.

Furthermore, the FEM analysis with these parameters results in a total displacement $2 \Delta f_{\mathrm{FEM}}=0.438 \mathrm{~mm}$. When entering the same parameters into (4) describing the theoretical model, the total displacement results in $2 \Delta f_{\text {theory }}=$ $0.569 \mathrm{~mm}$.

\subsection{Characterization of the Manufactured Grip Force Sensor.} For the characterization of the manufactured grip force sensor (Figure 5), an industrial material testing machine (Zwick 1456, Zwick Roell, Ulm, Germany) equipped with a load cell (Series K, $20 \mathrm{kN}$ nominal force, GTM Testing and Metrology, Bickenbach, Germany) was used to apply controlled force profiles to the grip force sensor. In order to equally distribute the load on the whole handle, the grip force sensor was placed between two custom-manufactured concave aluminum counterparts. In order to determine the characteristics of the grip force sensor, different cyclic and step force profiles were performed. The profiles of the applied force $F$ and resulting grip force sensor amplifier voltage output $U$ were recorded by the software of the material testing machine and by a 12-bit-resolution data acquisition card (USB 6008, National Instruments, Austin, TX, USA) connected to a computer running LabVIEW 2015 (National Instruments, Austin, TX, USA), respectively. The signals were synchronized and resampled at $1 \mathrm{kHz}$, and the voltage offset (for zero force) was always removed prior to subsequent data analysis. All

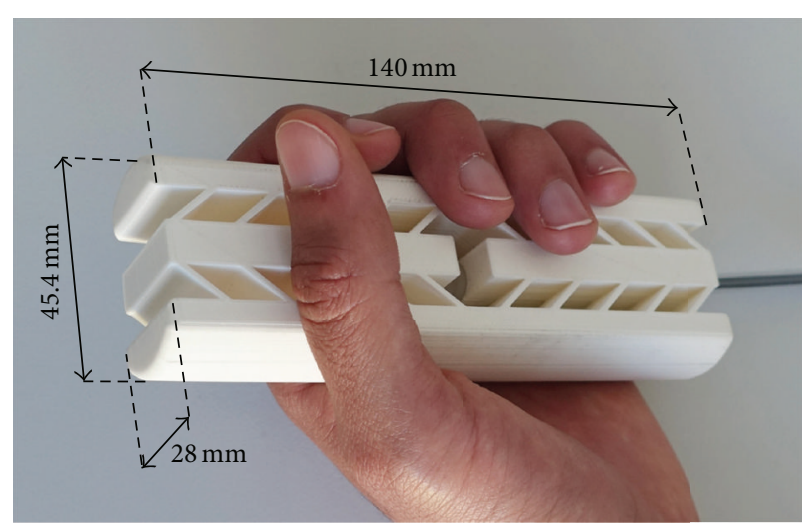

Figure 5: The developed grip force sensor. Total length $140 \mathrm{~mm}$, height $45.4 \mathrm{~mm}$ (with a curved surface of radius $22.7 \mathrm{~mm}$ ), and width $28 \mathrm{~mm}$. The total weight of the sensor is $140 \mathrm{~g}$.

analyses were performed in MATLAB R2014a (MathWorks, Natick, MA, USA).

In order to determine the transfer function, sensitivity, nonlinearity, resolution, nonrepeatability, and hysteresis, a force sweep with six consecutive trapezoidal cycles, from zero to the designed full range $800 \mathrm{~N}$, was performed. The whole force sweep (six cycles) lasted $10 \mathrm{~min}$. Using the leastsquare best-fit straight line (LS BFSL) method, the mean and standard deviation (SD) of the absolute and relative nonlinearity in $\mathrm{V}$ and in percentage to the full scale output (FSO), respectively, were computed for the twelve cycle parts (up and down parts of one cycle are considered as two separate parts). Since the nonlinearity depends on the force range (on which the characterization is based), which in turn depends on the specific application the grip force sensor is used for, the mean and SD of the nonlinearity were calculated as a function of the force range maximum at which the data was truncated before fitting the LS BFSL. The sensitivity $S_{0}$ in V/N was accordingly defined as the slope of the LS BFSL. In order to calculate the resolution $\Delta F_{\text {min }}=U_{\text {noise }} / S_{0}$, the noise level $U_{\text {noise }}$ was calculated by taking the standard deviation of the output voltage signal of the amplifier over $1 \mathrm{~s}$ when no load was applied to the grip force sensor. Absolute $(\mathrm{V})$ and relative (\%) (divided by the FSO) nonrepeatability consisted of the maximum output difference between up parts of consecutive cycles $(1-2,2-3, \ldots, 5-6)$. Mean and SD were calculated for the five cycle pairs. The nonrepeatability was also calculated as a function of an application force range maximum. The relative hysteresis (\%) was described by the maximum output difference between up and down cycle parts for a complete cycle to $800 \mathrm{~N}$ divided by the FSO. Again, mean and SD were calculated across the six cycles. Since the hysteresis requires a complete cycle, the hysteresis could not be computed for a varying application force range maximum. By calculating the root mean square (RMS) of the nonlinearity, the hysteresis, and the nonrepeatability in percentages, the RMS accuracy (\%) was calculated. The above characterization was repeated for the hysteresis of a force cycle profile going up to $500 \mathrm{~N}$, only. 
Creep and recovery were examined by loading the grip force sensor with $800 \mathrm{~N}$ for $30 \mathrm{~min}$ and removing the complete load during the following $60 \mathrm{~min}$. By taking the derivative of the estimated output force (i.e., of the output voltage $U$ times the sensitivity $S_{0}$ ), the creep and recovery rates were visualized. After this long-term load measurement, the characterization with cycles up to $800 \mathrm{~N}$ was repeated to investigate, whether the long-term loading had a nonnegligible effect on the grip force sensor properties. Since the compliant structure and fiber-optic sensor concepts should allow for very dynamic behavior and fast responses to rapid force applications, force steps from $0 \mathrm{~N}$ to $500 \mathrm{~N}$ within $0.2 \mathrm{~s}$, as well as from $500 \mathrm{~N}$ back to $0 \mathrm{~N}$, were applied on the grip force sensor. The estimated output force was compared to the force step profile of the material testing machine.

2.5. Experimental Pilot Study. In order to test the developed grip force sensor, a pilot experiment with one healthy male subject (aged 27) performing a motor task inside, as well as outside, an MRI scanner was conducted.

In both conditions, the subject held the grip force sensor with the dominant hand using a power grip. During the motor task, the subject was instructed to generate a precise grip force level as displayed on a screen by a target area $( \pm 10 \%$ of the required force) on a vertical thermometer bar indicator. The level of the thermometer represented the subject's applied grip force in real time. The subject was required to hold the grip force within the target area for $4 \mathrm{~s}$. A set of different target forces $(\epsilon\{100,200,300,400\} N)$ was presented in randomized order, and each force level was repeated six times.

In the first condition, the subject was lying in a running Philips Ingenia 3.0 T MRI scanner (Philips, Amsterdam, Netherlands) located at cereneo AG, Vitznau, Switzerland. A 32-channel Philips dStream head coil was used. The visual feedback was displayed on a screen placed at the end of the scanner bore, visible to the subject through a mirror mounted on the head coil. While performing the motor task, one run of functional data in a gradient echo $\mathrm{T} 2{ }^{*}$-weighted echo-planar image was acquired. In the second condition, the subject underwent the same protocol but was seated at a table outside the scanner room and the visual feedback was displayed on a monitor placed in front of the subject. Each condition lasted for about $5 \mathrm{~min}$. Between the two conditions, the subject could rest for $30 \mathrm{~min}$ in order to avoid carryover effects due to physical and mental fatigue.

The experimental protocol and visual feedback were implemented in LabVIEW 2015 (National Instruments, Austin, TX, USA), as well as the acquisition of the grip force sensor output using a 12-bit-resolution data acquisition card (USB 6008, National Instruments, Austin, TX, USA). Prior to each condition, the sensitivity and offset of the grip force sensor were calibrated-simply by using no load and by placing a weight of $4.5 \mathrm{~kg}$ on the grip force sensor lying in the concave aluminum counterparts, in order to minimize effects due to viscoelasticity discussed in Section 4. The raw force data was preprocessed by using a second-order low-pass Butterworth filter with a cut-off frequency of $3 \mathrm{~Hz}$. The mean and the standard deviation were computed for the last $3 \mathrm{~s}$ of each trial. In order to demonstrate statistical equivalence between the session inside and session outside the MRI scanner the "two one-sided tests" (TOST) procedure [31] was conducted. The equivalence boundary for the means of the measured values was defined to be $\pm 5 \%$ of the target value $( \pm 5 \mathrm{~N}$, $\pm 10 \mathrm{~N}, \pm 15 \mathrm{~N}$, and $\pm 20 \mathrm{~N}$ ). The equivalence boundary for the standard deviations of the measured values was defined to be $\pm 3 \mathrm{~N}$, regardless of the target value. Given that these equivalence boundaries are smaller than Weber's constant for just noticeable difference in weight perception [32], it can be assumed that people will not likely be able to distinguish whether the force they produced differed within this error margin. This means that the assumptions of statistical equivalence should also translate to experienced equivalence by a participant. For all statistical test, the significance level was set to $\alpha=0.05$. The data analysis was performed in MATLAB R2014a (MathWorks, Natick, MA, USA).

\section{Results}

3.1. Characterization of the Manufactured Grip Force Sensor. The transfer function of the grip force sensor, as well as absolute nonlinearity, sensitivity, absolute resolution, and absolute nonrepeatability as a function of an application-specific force range maximum, is presented in Figure 6 for the force sweep cycles up to $800 \mathrm{~N}$ before and after long-term load. The results revealed a very high linearity when basing the characteristics on force ranges up to $500 \mathrm{~N}$ (relative nonlinearity below $2.5 \%)$. Furthermore, "noisy" characteristics can be seen as a function of the application-specific force range maximum for small force ranges, which is most likely due to the limited number of data points for the LS BFSL fit, thus also visible in sensitivity and resolution. The noise level was $0.0019 \mathrm{~V}$, approximatively, corresponding to around $2 \mathrm{~N}$. The complete characteristics for the force ranges of $800 \mathrm{~N}$ and $500 \mathrm{~N}$ are presented in Table 1. For both force ranges, both the relative nonrepeatability and relative hysteresis were around $1 \%$ or less, and the accuracy was at $5.99 \%$ for $800 \mathrm{~N}$ and $1.57 \%$ for $500 \mathrm{~N}$. Moreover, the sensitivity for $800 \mathrm{~N}$ was marginally lower being $0.843 \mathrm{mV} / \mathrm{N}$ compared to $0.978 \mathrm{mV} / \mathrm{N}$ for $500 \mathrm{~N}$. The creep and recovery behavior for the long-term load of $800 \mathrm{~N}$ is visualized in Figure 7 showing the applied force and the estimated output force based on the sensor reading, as well as its derivative. The derivative shows an exponential behavior reaching steady state asymptotically. At $30 \mathrm{~min}$ of continuous load, the sensor still presented an output creep rate of $1 \mathrm{~N} / \mathrm{min}$, and after $60 \mathrm{~min}$ of recovery without load, it presented a rate of $-0.2 \mathrm{~N} / \mathrm{min}$ and an estimation error of $26.5 \mathrm{~N}$. The characterization of the grip force sensor after this long-term load is presented together with the pre-longterm load characterization in Figure 6, which shows high correspondence between the two conditions. The behavior of the grip force sensor when a force step $(0-500 \mathrm{~N}$ within $0.2 \mathrm{~s})$ is applied is shown in Figure 8. The measured force follows the applied force with no noticeable time delay.

3.2. Experimental Pilot Study. The comparison of the force means and standard deviations for the two measurement conditions inside and outside the MRI scanner is illustrated in Figure 9. Statistical analyses of the force averages revealed 
TABLE 1: Complete grip force sensor characteristics for the force ranges $800 \mathrm{~N}$ and $500 \mathrm{~N}$.

\begin{tabular}{|c|c|c|c|c|c|c|}
\hline Application & $\begin{array}{l}\text { Sensitivity } \\
(\mathrm{mV} / \mathrm{N})\end{array}$ & $\begin{array}{l}\text { Nonlinearity } \\
\text { (LS BFSL) (\%) }\end{array}$ & Nonrepeatability (\%) & Hysteresis (\%) & Resolution (N) & $\begin{array}{l}\text { Accuracy } \\
\text { (RMS) (\%) }\end{array}$ \\
\hline $800 \mathrm{~N}$ & $0.843 \pm 0.003$ & $10.33 \pm 0.10$ & $0.76 \pm 0.38$ & $0.72 \pm 0.38$ & $2.219 \pm 0.008$ & 5.99 \\
\hline $500 \mathrm{~N}$ & $0.978 \pm 0.005$ & $2.37 \pm 0.22$ & $1.02 \pm 0.50$ & $0.85 \pm 0.09$ & $1.911 \pm 0.009$ & 1.57 \\
\hline
\end{tabular}
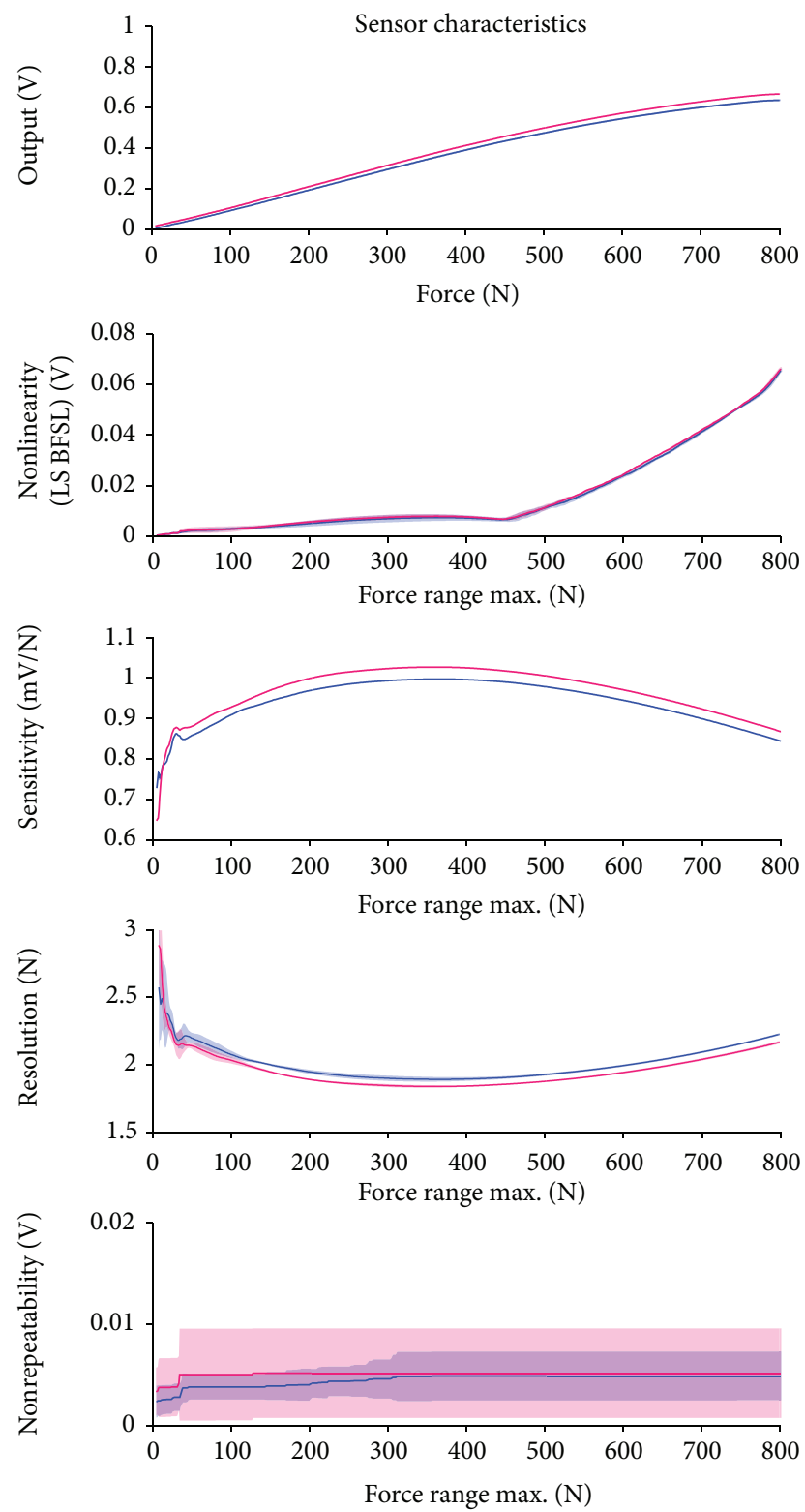

Before long-term load

$$
\begin{aligned}
& \text { Mean } \\
& \text { SD }
\end{aligned}
$$$$
\text { After long-term load }
$$$$
\text { - Mean }
$$$$
\text { SD }
$$

FIGURE 6: Characteristics of the grip force sensor determined in a force sweep from zero to $800 \mathrm{~N}$ for both, before (blue) and after (red) $800 \mathrm{~N}$ long-term load: transfer function, absolute nonlinearity, sensitivity, absolute resolution, and absolute nonrepeatability. Except for the transfer function, all characteristics are visualized as a function of a maximum force range, on which the characterization is based. Depending on the required force range for a specific application, the characteristics can be read off.
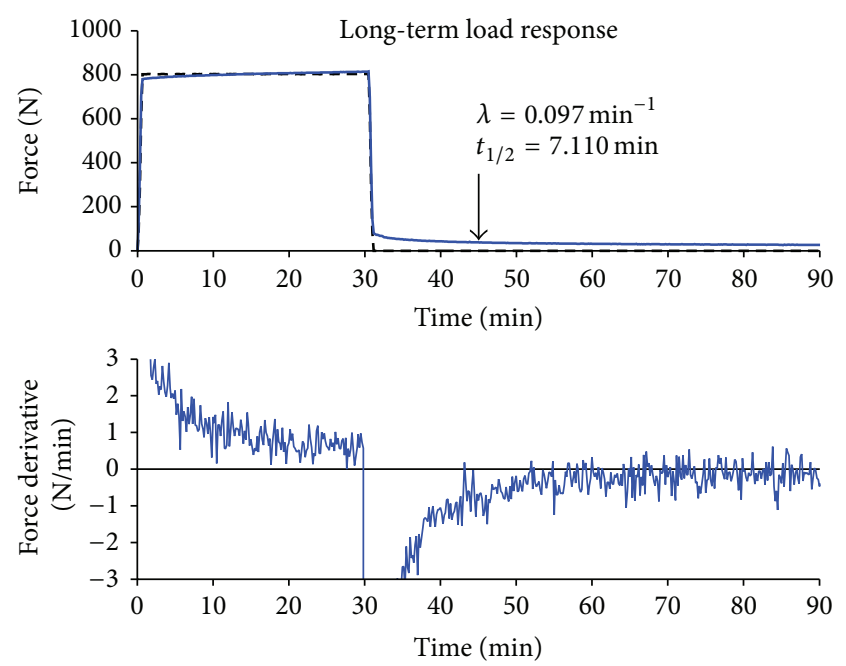

FIGURE 7: Creep and recovery response following a long-term load of $800 \mathrm{~N}$. The dashed line represents the applied force by the material testing machine, and the solid line represents the estimated force, as well as its derivative, based on the sensor reading and sensitivity. The decay rate $\lambda$ and the half-life $t_{1 / 2}$ are provided for the exponential drift of the force after the long-term load.

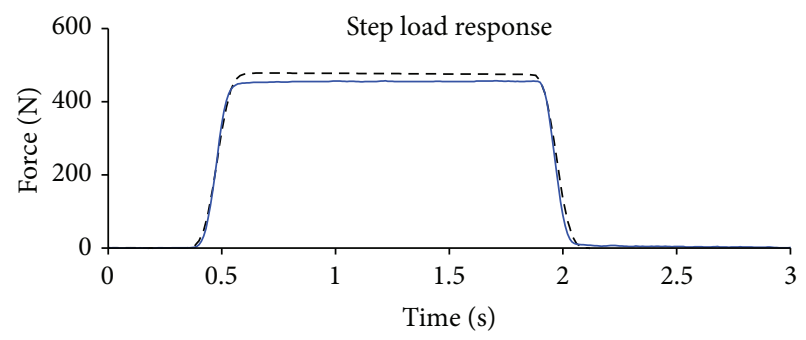

Figure 8: Grip force sensor response to a force step from 0 to $500 \mathrm{~N}$ within $0.2 \mathrm{~s}$ and from 500 to $0 \mathrm{~N}$. The dashed line represents the applied force by the material testing machine, and the solid line represents the estimated force based on the sensor reading and sensitivity.

that both conditions can be considered as being equivalent with $p_{\text {lower }}<0.003$ and $p_{\text {upper }}<0.0007$ for the lower and upper equivalence boundaries for all four investigated reference values $\left(90 \% \mathrm{CI}_{\text {lower }}>-2.37 \%\right.$ and $90 \% \mathrm{CI}_{\text {upper }}<2.67 \%$ with respect to the specific target values). Furthermore, the comparison of the force standard deviations showed the same trend, with statistical equivalence for all four investigated target values ( $p_{\text {lower }}<0.016, p_{\text {upper }}<0.013$ and $90 \% \mathrm{CI}_{\text {lower }}>$ $\left.-2.37 \mathrm{~N}, 90 \% \mathrm{CI}_{\text {upper }}<2.24 \mathrm{~N}\right)$. 


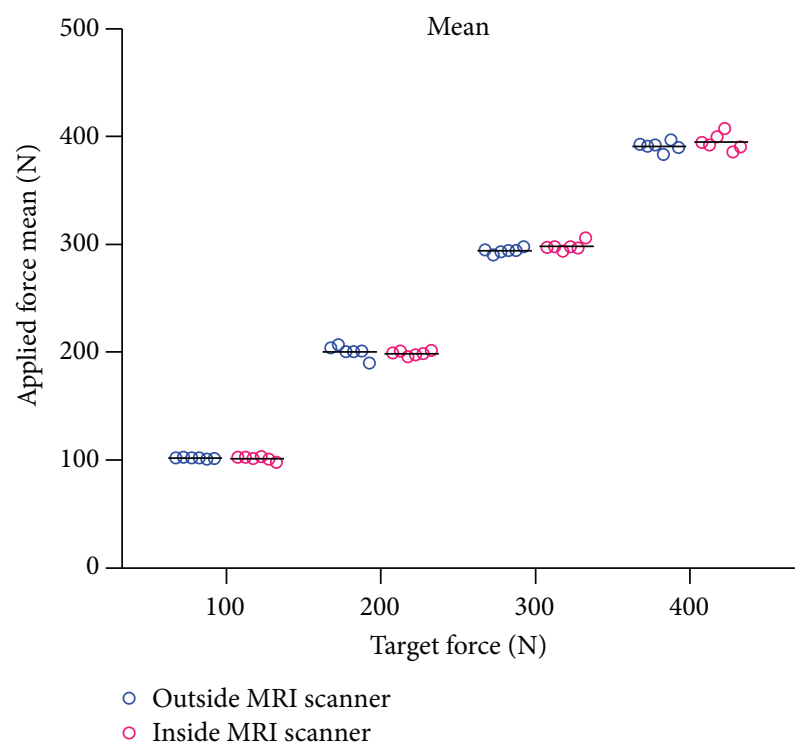

(a)

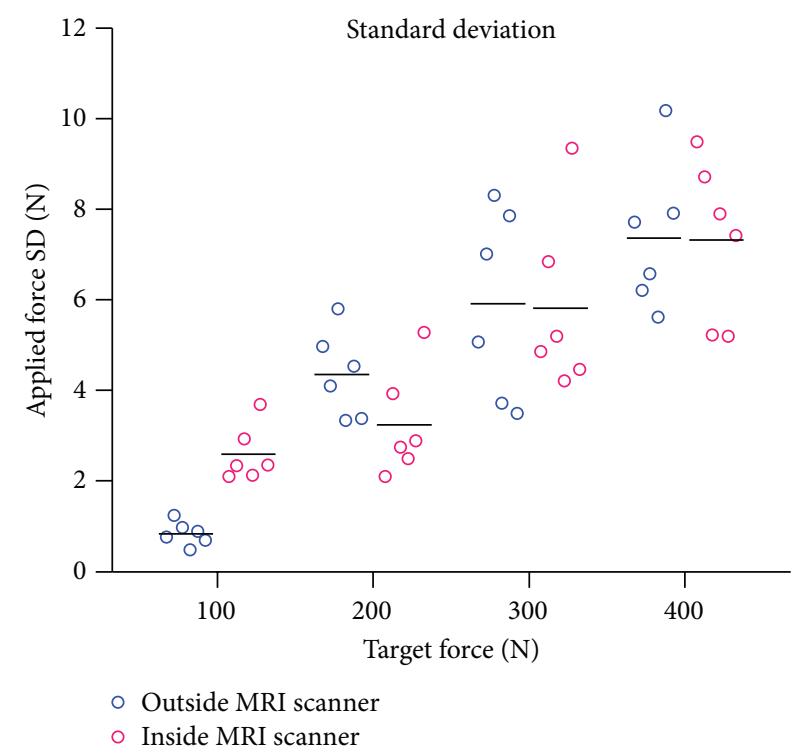

(b)

FIGURE 9: The outcome measures force mean (a) and standard deviation (SD) (b) for the comparison of the two conditions inside and outside of the MRI scanner are presented for each target value separately. Note that, according to the statistical tests, both conditions can be considered as being equivalent regardless of the outcome measure.

\section{Discussion}

This paper proposes an easily customizable, inherently MRIcompatible grip force sensor composed of a commercial intensity-based fiber-optic distance sensor and a compliant structure printed with a low-cost $3 \mathrm{D}$ printer. The comprehensive characterization of the grip force sensor showed that it can be safely used for measurements up to $800 \mathrm{~N}$, which is comparable to the force range of commercially available grip force sensors (e.g., Biopac Systems TSD121C). The range 0$500 \mathrm{~N}$ shows the best properties with a very high linearity (nonlinearity of $2.37 \%$ ) and accuracy of $1.57 \%$, comparable to commercially available products (e.g., $1 \%$ for the Biopac Systems, ADInstruments MLT004/ST).

When comparing more closely the characteristics of the proposed grip force sensor for a force range of $500 \mathrm{~N}$ and $800 \mathrm{~N}$, the only significant difference is the nonlinearity, since the slope, that is, sensitivity, of the transfer function decreases with increasing forces. This decrease in sensitivity (as well as the increase in sensitivity in the range of $100-300 \mathrm{~N}$ ) is most probably attributable to the increasing nonlinearity of the fiber-optic sensor itself. In order to obtain a complete characterization including hysteresis not only for the maximal range $(0-800 \mathrm{~N})$ but also for the more linear range $(0-500 \mathrm{~N})$, the characterization was repeated for a force cycle profile going up to $500 \mathrm{~N}$, only.

The characterization showed minimal hysteresis and nonrepeatability during nominal use. The sensor characteristics, such as linearity and sensitivity, are only marginally affected by the long-term load and, therefore, the sensor will deliver a repeatable output. Yet, during long-term load, the sensor presented some creep and a relatively slow recovery, as previously shown in similar research $[24,33]$. These characteristics were expected due to the viscoelastic properties of PLA and are accepted as a trade-off for the inherent MRI compatibility of the material and the customizability enabled by the fabrication method. Furthermore, models to account for these effects and minimize the measurement error have been proposed $[33,34]$. Also, it should be noted that a load of $800 \mathrm{~N}$ lasting $30 \mathrm{~min}$ is an unlikely event in fMRI experiments involving grip force. Nevertheless, remaining steady-state errors (i.e., offset drift during use) can be eliminated by simply removing the offset after a determined amount of time, that is, by conducting a quick calibration as described in the experimental pilot study.

The results from the force step applied to the grip force sensor showed a good dynamic response of the sensor. Thus, it could be used for motor control tasks with rapidly changing forces and to assess reaction and force rise/decrease times, which is an advantage over most commercially available MRI-compatible force sensors relying on air pressure measurement.

As the proposed sensor consists entirely of nonmagnetic and, except for the optical head, nonconducting materials and the sensing is performed by reflected light intensity measurement, it is inherently MRI-compatible. The optical head is made from brass and might lead to deformations of the magnetic field. However, this effect is small and highly localized due to the low magnetic susceptibility of brass [10]. Furthermore, the proposed sensing principle has been successfully applied in a number of neuroscience studies [35-40]. For these reasons, mutual interactions between the sensor and the MRI environment can be excluded for the intended application (grasp force measurement in human fMRI studies) and no in-depth compatibility testing with phantoms was 
performed. The analysis of the experimental pilot study showed equivalent behavior of the grip force sensor inside and outside the MRI scanner (despite not controlling for identical grip position on the handle). This property can be of special value for experiments where the forces are normalized with the maximum voluntary contraction (MVC) force to make the results comparable between subjects. As the MVC force may depend on the ergonomic design of the handle, the ability to acquire the MVC measurements outside the scanner and force during the experiment with the same device is highly desired. Furthermore, using the same device simplifies the acquisition of data by eliminating, for example, additionally required conversions between two systems.

Moreover, based on theoretical model and FEM analysis, we proposed equations and a recommended dimension range in order to customize the grip force sensor for applications requiring different sensing ranges. The theoretical model and the FEM analysis correspond well (approximately 20\%) and therefore provide a cross-validation. The existing small difference (between the two analysis methods) in total displacement of the sensor head with respect to the mirror may be attributed to various assumptions made to simplify the model (perpendicular direction of force application, rigid outer bars, linear behavior of the hinges, and uniform distribution of the force).

For force ranges of $0-800 \mathrm{~N}$ the sensor characteristics can be obtained directly from the presented graphs. Yet, for applications requiring forces smaller than $100 \mathrm{~N}$, we recommend using the sensor characteristics of a force range of at least $100 \mathrm{~N}$. This is due to the fact that for low force ranges the relative nonlinearity of the sensor output is high (as the absolute nonlinearity is divided by a smaller number, i.e., force range) and the number of data points available for the LS BFSL fit is limited. As a consequence, there are large variations in the slope of the LS BFSL fit (i.e., sensitivity) and the resolution for low force ranges. In order to obtain more precise measurements with the presented grip force sensor for forces beyond $500 \mathrm{~N}$, the transfer function can be described with a higher-order parametric function, or a lookup table of the nonapproximated transfer function can be used, in order to take the increasing nonlinearity into account. For other force ranges or higher SNR requirements in specific applications, the dimensions of the compliant structure can be adjusted to optimally match the fiber-optic sensor operation range. With the theoretical model and the parametric model derived from the FEM analyses, adapting the grip force sensor to a specific force range becomes very simple and can be done by primarily changing the hinge thickness. However, change of the hinge thickness may require a full characterization with the modified compliant structure and should be done within the permissible dimension range. Note that the lower end of the permissible dimension range is limited not only by internal stress but also by 3D-printer resolution. Nevertheless, for the presented designs the printer resolution of stateof-the-art low-cost 3D printers (in the range of $0.4 \mathrm{~mm}$ ) is largely sufficient and the structure can be printed accurately. Furthermore, today's 3D-printing technology allows simple manufacturing of custom ergonomic shapes. Thus, grooves on the handle could be added, in order to make the subjects' grip type and location more consistent or to adapt it to different hand sizes.

The grip force sensor presented in this paper can be fabricated within less than 24 hours requiring only access to a FDM 3D printer. Furthermore, the total material cost (including fiber-optic sensor, amplifier, and 3D-printed structure) is less than $\$ 300$.

\section{Conclusion}

This paper presented the design and characterization of an inherently MRI-compatible, 3D-printed grip force sensor, based on a commercially available intensity-based fiber-optic sensor and a compliant structure used to transduce force into displacement. The sensor can be fabricated with any low-cost FDM 3D printer within one day and provides accurate MRIcompatible grip force measurement covering a high range of forces at very affordable cost. Researchers can reproduce the presented design and use the sensor according to reported characteristics or adapt the sensing range to custom applications by changing a single parameter of the 3D-printed structure. The characteristics, affordability, and customizability make this sensor a valuable tool for the scientific study of motor control, decision-making, and related topics within and outside the MRI scanner.

\section{Competing Interests}

The authors declare that there are no competing interests regarding the publication of this paper.

\section{Authors' Contributions}

Tobias L. Bützer and Mike D. Rinderknecht contributed equally to this work.

\section{Acknowledgments}

The authors would like to thank D. Woolley and U. Baumgartner for their valuable input regarding the first designs, A. Luft and K. Lutz from cereneo AG for providing access to the MRI scanner, J. Snedeker for allowing the use of his measurement infrastructure, and T. Vögeli for his help with the measurements. This research was supported by the ETH Zurich Foundation in collaboration with Hocoma AG, by the ETH Research Grant ET-17 13-2, as well as by the Swiss National Science Foundation through the National Center of Competence in Research (NCCR) on Robotics.

\section{References}

[1] S. Ogawa, T.-M. Lee, A. R. Kay, and D. W. Tank, "Brain magnetic resonance imaging with contrast dependent on blood oxygenation," Proceedings of the National Academy of Sciences of the United States of America, vol. 87, no. 24, pp. 9868-9872, 1990.

[2] M. Kawato, T. Kuroda, H. Imamizu, E. Nakano, S. Miyauchi, and T. Yoshioka, "Internal forward models in the cerebellum: fMRI study on grip force and load force coupling," Progress in Brain Research, vol. 142, pp. 171-188, 2003. 
[3] N. S. Ward and R. S. J. Frackowiak, "Carbon 'quantum' dots for optical bioimaging," Brain, vol. 126, no. 4, pp. 873-888, 2003.

[4] C. Konrad, A. Jansen, H. Henningsen et al., "Subcortical reorganization in amyotrophic lateral sclerosis," Experimental Brain Research, vol. 172, no. 3, pp. 361-369, 2006.

[5] N. S. Ward, M. M. Brown, A. J. Thompson, and R. S. J. Frackowiak, "Neural correlates of motor recovery after stroke: A Longitudinal fMRI Study," Brain, vol. 126, no. 11, pp. 2476-2496, 2003.

[6] M. N. Hartmann, O. M. Hager, P. N. Tobler, and S. Kaiser, "Parabolic discounting of monetary rewards by physical effort," Behavioural Processes, vol. 100, pp. 192-196, 2013.

[7] M. Pessiglione, L. Schmidt, B. Draganski et al., "How the brain translates money into force: a neuroimaging study of subliminal motivation," Science, vol. 316, no. 5826, pp. 904-906, 2007.

[8] D. Chapuis, R. Gassert, L. Sache, E. Burdet, and H. Bleuler, "Design of a simple MRI/fMRI compatible force/torque sensor," in Proceedings of the IEEE/RSJ International Conference on Intelligent Robots and Systems (IROS '04), vol. 3, pp. 2593-2599, September-October 2004.

[9] N. Yu, R. Gassert, and R. Riener, "Mutual interferences and design principles for mechatronic devices in magnetic resonance imaging," International Journal of Computer Assisted Radiology and Surgery, vol. 6, no. 4, pp. 473-488, 2011.

[10] R. Gassert, D. Chapuis, H. Bleuler, and E. Burdet, "Sensors for applications in magnetic resonance environments," IEEE/ASME Transactions on Mechatronics, vol. 13, no. 3, pp. 335-344, 2008.

[11] G. R. Sutherland, I. Latour, and A. D. Greer, "Integrating an image-guided robot with intraoperative MRI," IEEE Engineering in Medicine and Biology Magazine, vol. 27, no. 3, pp. 59-65, 2008.

[12] J. Hidler, T. Hodics, B. Xu, B. Dobkin, and L. G. Cohen, "MR compatible force sensing system for real-time monitoring of wrist moments during fMRI testing," Journal of Neuroscience Methods, vol. 155, no. 2, pp. 300-307, 2006.

[13] J. Z. Liu, T. H. Dai, T. H. Elster, V. Sahgal, R. W. Brown, and G. H. Yue, "Simultaneous measurement of human joint force, surface electromyograms, and functional MRI-measured brain activation," Journal of Neuroscience Methods, vol. 101, no. 1, pp. 49-57, 2000.

[14] D. M. Krainak, T. B. Parrish, and J. P. A. Dewald, "A method to capture six-degrees-of-freedom mechanical measurements of isometric shoulder and elbow torques during event-related fMRI," Journal of Neuroscience Methods, vol. 161, no. 2, pp. 314322, 2007.

[15] H. Song, K. Kim, and J. Lee, "Development of optical fiber Bragg grating force-reflection sensor system of medical application for safe minimally invasive robotic surgery," Review of Scientific Instruments, vol. 82, no. 7, Article ID 074301, 2011.

[16] R. Monfaredi, R. Seifabadi, G. Fichtinger, and I. Iordachita, "Design of a decoupled MRI-compatible force sensor using fiber Bragg grating sensors for robot-assisted prostate interventions," in Proceedings of the Medical Imaging 2013: Image-Guided Procedures, Robotic Interventions, and Modeling, SPIE Medical Imaging, International Society for Optics and Photonics, February 2013.

[17] K. M. Moerman, A. M. J. Sprengers, A. J. Nederveen, and C. K. Simms, "A novel MRI compatible soft tissue indentor and fibre Bragg grating force sensor," Medical Engineering \& Physics, vol. 35, no. 4, pp. 486-499, 2013.

[18] M. Tada, S. Sasaki, and T. Ogasawara, "Development of an optical 2-axis force sensor usable in MRI environments," in
Proceedings of the 1st IEEE International Conference on Sensors (IEEE Sensors '02), vol. 2, pp. 984-989, Orlando, Fla, USA, June 2002.

[19] F. Taffoni, D. Formica, P. Saccomandi, G. Di Pino, and E. Schena, "Optical fiber-based MR-compatible sensors for medical applications: an overview," Sensors, vol. 13, no. 10, pp. 14105-14120, 2013.

[20] G. Berkovic and E. Shafir, "Optical methods for distance and displacement measurements," Advances in Optics and Photonics, vol. 4, no. 4, pp. 441-471, 2012.

[21] J. Arata, S. Terakawa, H. Fujimoto, J. Sulzer, and R. Gassert, "MRI-compatible grasping force sensor with an inclined double parallel structure using fiber optics," in Proceedings of the International Symposium on Flexible Automation (ASME/ISCIE '12), pp. 77-82, St. Louis, Mo, USA, June 2012.

[22] H. Xie, A. Jiang, H. A. Wurdemann, H. Liu, L. D. Seneviratne, and K. Althoefer, "Magnetic resonance-compatible tactile force sensor using fiber optics and vision sensor," IEEE Sensors Journal, vol. 14, no. 3, pp. 829-838, 2014.

[23] R. Riener, T. Villgrattner, R. Kleiser, T. Nef, and S. Kollias, "fMRI-compatible electromagnetic haptic interface," in Proceedings of the IEEE 27th Annual International Conference of the Engineering in Medicine and Biology Society (EMBS '05), pp. 7024-7027, Shanghai, China, September 2006.

[24] T. Butzer, B. Vigaru, and R. Gassert, "Design and evaluation of a compact, integrated fMRI-compatible force sensor printed by additive manufacturing," in Proceedings of the 10th IEEE World Haptics Conference (WHC '15), pp. 158-164, Evanston, Ill, USA, June 2015.

[25] S. B. Kesner and R. D. Howe, "Design principles for rapid prototyping forces sensors using 3-D printing," IEEE/ASME Transactions on Mechatronics, vol. 16, no. 5, pp. 866-870, 2011.

[26] U.-X. Tan, B. Yang, R. Gullapalli, and J. P. Desai, “Triaxial MRI-compatible fiber-optic force sensor," IEEE Transactions on Robotics, vol. 27, no. 1, pp. 65-74, 2011.

[27] NASA, Man-Systems Integration Standards. Volume I, Section 4. Human Performance Capabilities, 2008.

[28] K. Shinyama and S. Fujita, "Study on the electrical properties of a biodegradable plastic," in Proceedings of the 7th International Conference on Properties and Applications of Dielectric Materials, vol. 2, pp. 707-710, IEEE, Nagoya, Japan, June 2003.

[29] F. Rezgui, M. Swistek, J. M. Hiver, C. G'Sell, and T. Sadoun, "Deformation and damage upon stretching of degradable polymers (PLA and PCL)," Polymer, vol. 46, no. 18, pp. 7370-7385, 2005.

[30] S. Henein, Conception des Guidages Flexibles, PPUR Presses Polytechniques, 2001.

[31] S. J. Richter and C. Richter, "A method for determining equivalence in industrial applications," Quality Engineering, vol. 14, no. 3, pp. 375-380, 2002.

[32] C. E. Seashore, Elementary Experiments in Psychology, Weber's Law, Henry Holt and Company, New York, NY, USA, 1908.

[33] N. Kumar, O. Piccin, L. Meylheuc, L. Barbé, and B. Bayle, "Design and modeling of a polymer force sensor," IEEE/ASME Transactions on Mechatronics, vol. 21, no. 1, pp. 555-564, 2016.

[34] F. Parietti, G. Baud-Bovy, E. Gatti, R. Riener, L. Guzzella, and H. Vallery, "Series viscoelastic actuators can match human force perception," IEEE/ASME Transactions on Mechatronics, vol. 16, no. 5, pp. 853-860, 2011.

[35] M. P. Vlaar, W. Mugge, P. F. Groot et al., "Targeted brain activation using an MR-compatible wrist torque measurement 
device and isometric motor tasks during functional magnetic resonance imaging," Magnetic Resonance Imaging, vol. 34, no. 6, pp. 795-802, 2016.

[36] M. L. Blefari, J. Sulzer, M.-C. Hepp-Reymond, S. Kollias, and R. Gassert, "Improvement in precision grip force control with selfmodulation of primary motor cortex during motor imagery," Frontiers in Behavioral Neuroscience, vol. 9, article 18, 2015.

[37] M. Haruno, G. Ganesh, E. Burdet, and M. Kawato, "Differential neural correlates of reciprocal activation and cocontraction control in dorsal and ventral premotor cortices," Journal of Neurophysiology, vol. 107, no. 1, pp. 126-133, 2012.

[38] J. S. Sulzer, V. S. Chib, M.-C. Hepp-Reymond, S. Kollias, and R. Gassert, "BOLD correlations to force in precision grip: an eventrelated study," in Proceedings of the 33rd Annual International Conference of the IEEE Engineering in Medicine and Biology Society (EMBS '11), pp. 2342-2346, September 2011.

[39] S. Haller, D. Chapuis, R. Gassert, E. Burdet, and M. Klarhöfer, "Supplementary motor area and anterior intraparietal area integrate fine-graded timing and force control during precision grip," European Journal of Neuroscience, vol. 30, no. 12, pp. 24012406, 2009.

[40] G. Ganesh, D. W. Franklin, R. Gassert, H. Imamizu, and M. Kawato, "Accurate real-time feedback of surface EMG during fMRI," Journal of Neurophysiology, vol. 97, no. 1, pp. 912-920, 2007. 


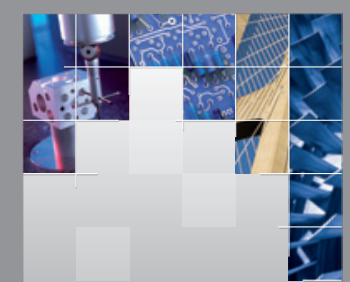

\section{Enfincering}
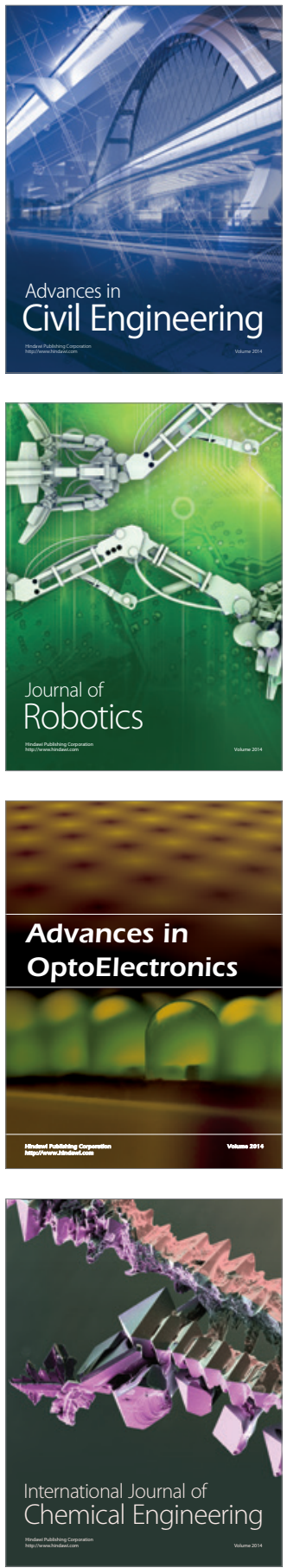

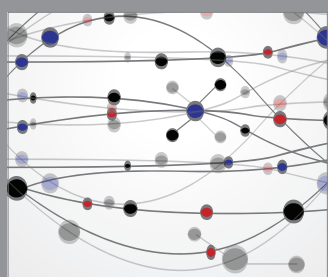

The Scientific World Journal

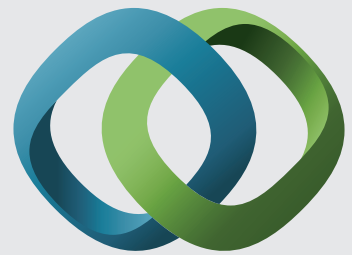

\section{Hindawi}

Submit your manuscripts at

http://www.hindawi.com
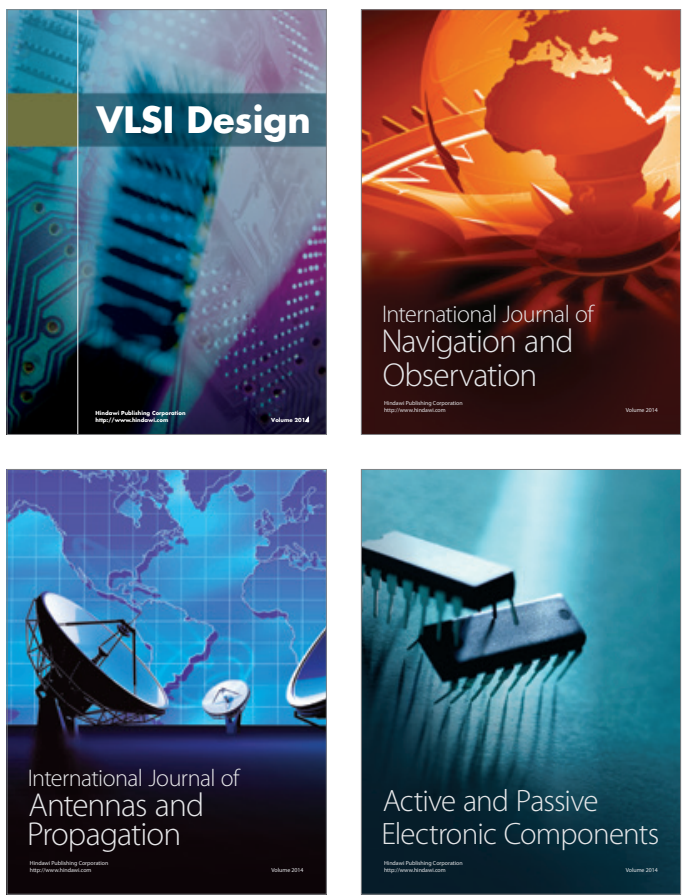
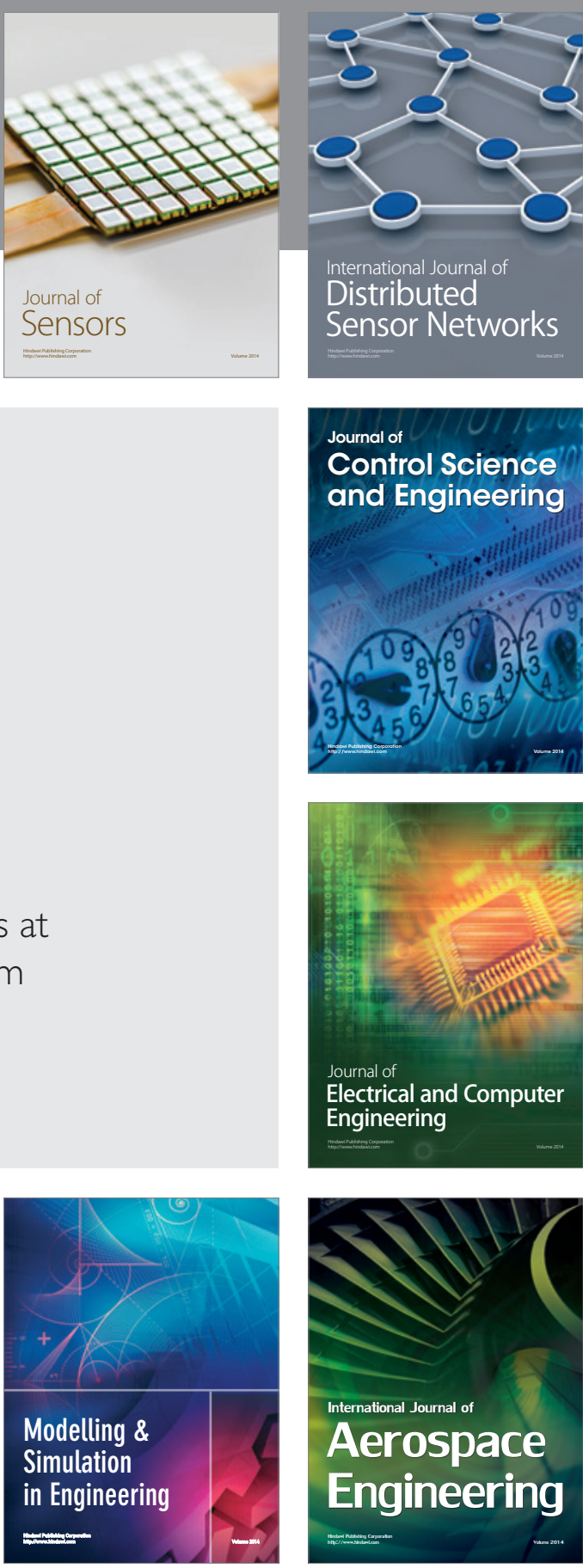

International Journal of

Distributed

Sensor Networks

Journal of

Control Science

and Engineering
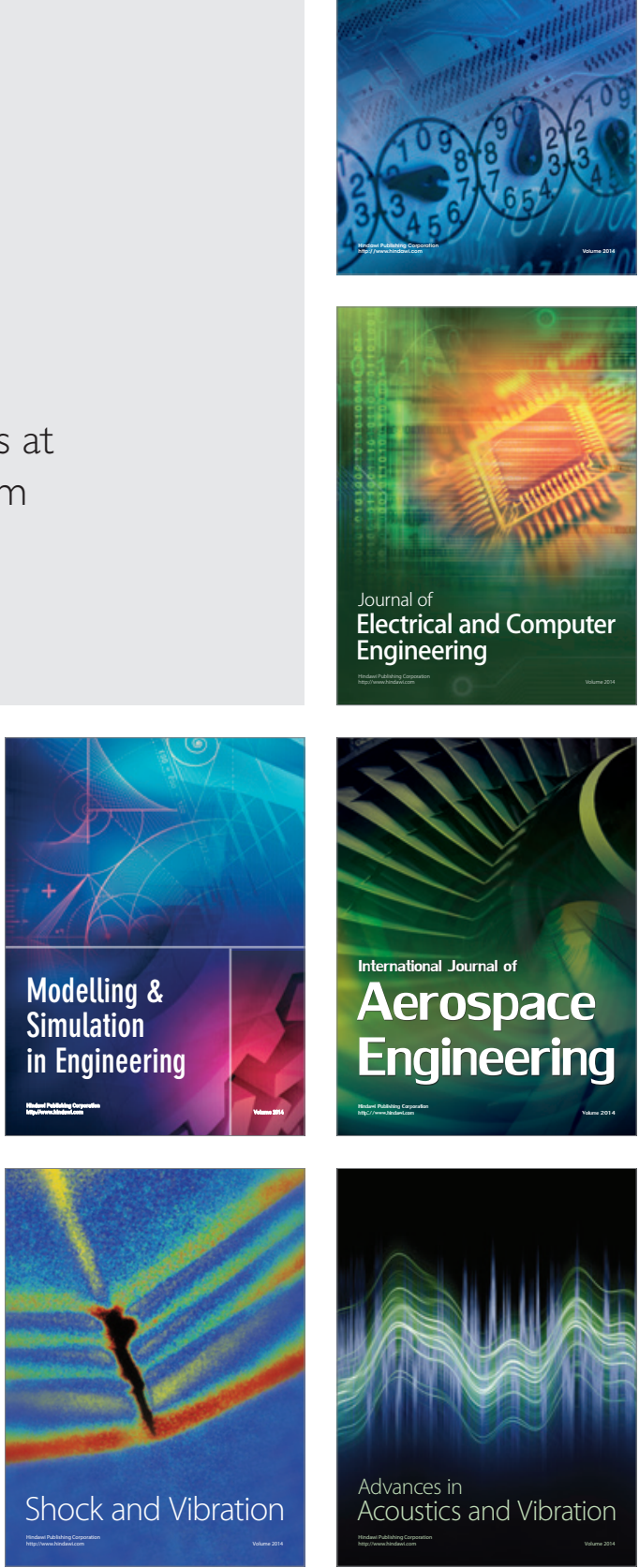